Mendioroz Lacambra, A.M. y Rivero Gracia, P. (2019). Componentes y dimensiones que caracterizan una buena praxis en Educación Infantil (0-3 años). Revista de Investigación Educativa, 37(1), 217-230.

DOI: http://dx.doi.org/10.6018/rie.37.1.318521

\title{
Componentes y dimensiones que caracterizan una buena praxis en Educación Infantil (0-3 años)
}

\section{Components and dimensions that characterize a good practice in Early Childhood Education (0-3 years)}

\author{
Ana María Mendioroz Lacambra* y Pilar Rivero Gracia** \\ *Universidad Pública de Navarra \\ ** Universidad de Zaragoza
}

\begin{abstract}
Resumen
Para mejorar la calidad educativa, es fundamental investigar y visibilizar buenas prácticas docentes. Desde hace varios años y en esta línea, las autoras intervienen en proyectos de innovación e investigación centrados en la Educación Infantil, lo que les ha permitido conocer y analizar la realidad educativa de esta etapa. Este artículo se centra en los resultados de un proyecto concreto de investigación, en el que participan universidades de ámbito europeo y latinoamericano con el objetivo de reconocer, analizar, normalizar, representar y hacer visibles 100 buenas prácticas en Educación Infantil, en un marco plural de características y condiciones. Se emplea una metodología cualitativa que permite vincular creencias implícitas con las prácticas docentes, y se recopilan los datos mediante entrevistas, observaciones en el aula y registros. En esta ocasión se presentan los criterios registrados por el grupo de investigación para reconocer buenas prácticas en el tramo 0-3 años, donde se evidencian, mediante las narrativas de las docentes, apoyadas por las observaciones y registros realizados en el aula, los componentes conceptuales y operacionales que caracterizan una buena praxis en Educación Infantil, en la línea de estudios precedentes.

Palabras clave: calidad de la educación; educación de la primera infancia; buenas prácticas; investigación cualitativa.
\end{abstract}

Correspondencia: Ana María Mendioroz Lacambra, anamaria.mendioroz@unavarra.es, Dpto. Geografía e Historia Universidad Pública de Navarra Campus de Arrosadía (31006). Pamplona, España. 


\begin{abstract}
To improve the educational quality, it is fundamental to investigate and make visible good teaching practices. For several years and in this line, the authors have intervened in innovation and research projects focused on early childhood education, which has allowed them to know and analyze the educational reality of this stage. Specifically this work that is now presented, is the result of a subsequent research project, in which European universities and Latin American universities participate, with the aim of detecting, analyzing, systematizing and representing 100 good practices in Early Childhood Education, in a framework of plural characteristics and conditions. A qualitative methodology is used to link assumed beliefs with teaching practices, and data are collected through interviews, observations in the classroom and records. This article presents some of the criteria proposed by the research group to identify good practices, in the 0-3year period, where they are evidenced, through the narratives of the teachers, supported by the observations and records made in the classroom, the conceptual and operative components that characterize a good praxis in Infantile Education.

Keywords: Educational quality; Early Childhood Education, best practices; qualitative research.
\end{abstract}

\title{
Introducción
}

El principal patrimonio con el que cuenta la humanidad es la educación. Durante la primera infancia se sientan las bases para el desarrollo posterior en todos los ámbitos de la vida (Bodero Cáceres, 2017), de ahí que la prioridad de los organismos responsables en educación es promover la investigación y la participación de los profesionales, con el objeto de reconocer, analizar, normalizar y representar buenas prácticas de atención a las criaturas, haciéndolas visibles a toda la comunidad educativa y a nivel mundial, con el objetivo de que sirvan de referencia en la mejora de las políticas educativas y en suma, en la calidad de la educación (Bureau des institutions démocratiques et des droits de l'homme de l'OSCE [BIDDH], 2011; Campos, 2013).

Son muchos los factores que intervienen en la calidad educativa, pero sin duda la práctica es uno de los más determinantes (Mir Pozo \& Ferret Ribot, 2014), ya que implica componentes éticos y orienta la actividad hacia la mejora de las situaciones y contextos en los que se considera. No existe un modelo de buena práctica aplicable a cualquier situación, porque las buenas prácticas se refieren a aquellas modalidades diversas de responder con eficacia y satisfacción a las diferentes demandas del contexto (Braslavsky, Abdoulaye \& Patiño 2003).

En educación se ha pasado de "los enfoques centrados en la calidad en términos absolutos, a otros basados en la identificación y visibilización de buenas prácticas [...] desde una perspectiva más inductiva y situada [...] lo bueno es aquello que funciona bien, aquello que es valorado por sus protagonistas [...] y es reconocido como valioso por los colegas, o por sus destinatarios indirectos" (Zabalza, 2012, p.18). El potencial de una buena práctica se sitúa en la práctica misma, por lo que para identificar las razones que la fundamentan es necesario evidenciar la intencionalidad que la sustenta y por supuesto contextualizarla (Hoyuelos, 2017; Zabalza, 2016). Por lo general y aunque priman los estudios que identifican variables características del buen profesor, de las buenas escuelas 
o de las estrategias en niveles fundamentalmente de educación superior (Larose, Grenon, Bourque \& Bédard, 2011), Lewin Benham (2011) investiga sobre las dimensiones que satisfacen los criterios de buenas prácticas en educación inicial, o Howes (2010), similar al que sustenta este artículo, se sitúa en la línea estratégica de reconocer, analizar, normalizar, representar y dar a conocer buenas prácticas. Como se puede comprobar en la etapa de Educación Infantil no se contemplan estudios nacionales o internacionales, basados en este tema. De hecho, en España son muy pocos los proyectos de investigación financiados que se han ocupado de analizar las dimensiones a tener en cuenta para satisfacer los criterios de buenas praxis, o de identificar las características que permiten atribuir esta categoría a una práctica, y solo el nuestro Grupo Interuniversitario de Estudios (GIE), se ha especializado durante los últimos años en el análisis de buenas prácticas a través de proyectos vinculados al Programa de Fomento de la Investigación Científica y Técnica de Excelencia (I+D), financiados por el Ministerio de Economía y Competitividad (Cid, Pérez \& Zabalza, 2013; Mendioroz \& Fiz, 2016) y ahora en Educación Infantil (Bartau Rojas, Azpillaga Larrea \& Joaristi Olariaga, 2017). Fruto de esta investigación es el trabajo de Denébola y Rodríguez (2016), sobre materiales didácticos.

Este artículo desde la experiencia en innovación e investigación educativa de las autoras, se basa concretamente en el proyecto de investigación «Diseño Curricular y Buenas Prácticas en Educación Infantil: una visión internacional, multicultural e interdisciplinar», enmarcado en el Plan Estatal de Investigación Científica y Técnica y de Innovación, dentro del Programa de Fomento de la Investigación Científica y Técnica de Excelencia (I+D), del Ministerio de Economía y Competitividad de España. Se plantea en un marco internacional con la participación de equipos de investigación de Portugal, Reino Unido, Italia, Chile, Argentina, México, Brasil y España, con las universidades de Santiago, Vigo, Coruña, Granada, Islas Baleares, La Laguna y la Pública de Navarra. Además, se enfoca desde la multiculturalidad, buscando modelos que respondan a las características propias del contexto cultural, y desde la multidisciplinariedad, analizando buenas prácticas en los diferentes modos de atención a las criaturas y en las diversas áreas curriculares. Tiene en cuenta las condiciones propuestas por el Bureau des Institutions Démocratiques et des Droits de l'Homme (BIDDH, 2011) para analizar buenas prácticas en la Educación Infantil: documentación institucional sobre cómo hay que llevar a cabo el proceso de enseñanza aprendizaje para considerarlo de calidad, las propias instituciones de la etapa, su organización, los recursos, etc. También los escenarios de aprendizaje, la formación del profesional que atiende a las criaturas, y todo ello de forma contextualizada e integradora en la comunidad educativa, con la vinculación de las familias. En el análisis se tiene en cuenta la propia práctica, la visión de los agentes implicados y la relación entre ambos (López de Maturana, 2012).

Se parte de la hipótesis de que la práctica educativa se ajusta a las creencias de quienes la realizan (Molina, Miralles, Deusdad \& Alfageme, 2017), estas ideas están en permanente evolución y se pueden recuperar mediante técnicas de ingeniería del conocimiento como son los mapas conceptuales. El repertorio que manejan los agentes con buenas prácticas educativas es reflexionado y ajustado a la realidad, y estas praxis se refuerzan y tienden a producirse en los contextos que las promueven; además, en ambientes sociales que otorgan gran importancia a la educación de la infancia y tienen en cuenta a toda la comunidad educativa (Lewin Benham, 2011). 
Emplea metodologías que facilitan la visibilización de las creencias asumidas epistemológicas, didácticas y actitudinales, por su importancia en la construcción de la identidad profesional (Barnett \& Di Napoli, 2007), y en las prácticas del profesorado, como son las historias de vida (Moriña, 2017) y la Teoría del posicionamiento (Davies \& Harré, 1990), aparato conceptual y metodológico adecuado para estudiar las vidas profesionales, ya que considera que toda interacción es narrativa, cambiante y contextual, empleado por vez primera en nuestro país en niveles básicos de enseñanza por Marcelo (1987) y Zabalza (1987). La investigación se completa con el diseño de un dispositivo informático que permite mediante un mapa conceptual, (Novak, 1998) visualizar las interconexiones de las ideas que subyacen en las prácticas estudiadas, y referirlas a prácticas concretas, con el objetivo último de construir una comunidad de aprendizaje e intercambio de experiencias a nivel internacional e interdisciplinar, constituida por investigadores y profesionales de la Educación Infantil.

\section{Objetivos}

En todos los equipos, nacionales e internacionales, que intervienen en este proyecto de investigación, se trabaja con tres objetivos:

1. Reconocer, analizar, normalizar, representar y hacer visibles un centenar de buenas prácticas teniendo en cuenta los diversos elementos y dimensiones que caracterizan a la Educación Infantil, empleando las narraciones de los docentes implicados, observaciones y registros realizados de la práctica.

2. Establecer un marco de características y condiciones plural y flexible sobre las experiencias analizadas, identificando qué tienen en común y de diferente, de propio y de transferible.

3. Generar grupos de trabajo a nivel internacional que faciliten el conocimiento y el trasvase de experiencias.

\section{Método}

\section{Población y Muestra}

Para construir la muestra se revisan normativas y estudios referidos a las condiciones y estándares que debe reunir una Educación Infantil de calidad, y se consulta a diferentes informantes conocedores de esta etapa a los que se les plantea la pregunta ¿qué experiencias destacarías en tu ciudad, zona o región y por qué?

Los criterios para seleccionar la muestra, comunes a todos los equipos de trabajo, se refieren a actuaciones bien valoradas por responsables políticos, técnicos o profesionales de la zona que supongan innovación respecto a la atención a los más pequeños con respuestas originales a necesidades de las familias y de los niños y niñas, su inserción en el entorno, su forma de trabajo con padres y madres; mejora en recursos, espacios, forma de trabajar, metodologías experimentadas con éxito que hayan creado materiales innovadores o generado ambientes de aprendizaje con características especiales; actividades que supongan una innovación o una forma diferente 
y original de trabajar; modos de relación, de agrupamiento; prácticas sostenidas por una fuerte fundamentación científica; estilo de documentar las actividades; iniciativas o instituciones evaluadas positivamente en alguna característica propia, escuelas inclusivas con prácticas destacables en la atención a las necesidades educativas especiales, educadores que se hayan destacado por sus ideas, sus escritos, sus intervenciones relacionados con la necesidad de transformar la Educación Infantil, y finalmente, que resulten satisfactorias para los agentes implicados. Sobre la base de estos criterios, y una vez realizado el muestreo con el objetivo de abarcar las distintas modalidades de Educación Infantil y todas las áreas curriculares, mediante un torbellino de ideas se propone un pool de iniciativas susceptibles de ser seleccionadas. Las informantes son profesoras, excepto en un caso, con una media de edad de 45 años. Por sus datos personales y vitales que han adjuntado a la encuesta, se trata de personas con una vocación muy temprana hacia la docencia, comprometidas con temas sociales desde su adolescencia, reflexivas y críticas con el sistema educativo. Todas ellas coinciden en la insatisfacción que sintieron al incorporarse a la vida laboral y comprobar que su práctica de aula no respondía a necesidades reales de la infancia. Además, reconocen que su frustración personal les condujo a conocer las escuelas Reggio Emilia, muchas de ellas a través del coordinador de las escuelas infantiles de Navarra.

El reparto de los casos se realiza en función del tamaño del equipo de cada universidad participante, con el objetivo de que cada investigador/a asuma el seguimiento de una práctica al año, además, los datos obtenidos se analizan por clusters y en general. Concretamente nuestro grupo de trabajo recibe el encargo de identificar y analizar 12 buenas prácticas realizadas en el entorno, 9 de ellas en el tramo 0-3 años, lo que representa el 30\% del total de las analizadas en esta categoría.

Teniendo en cuenta que no existe un modelo de buena práctica transferible a cualquier situación y contexto, el equipo establece, no obstante, como criterios teóricos básicos necesarios para una buena práctica en Educación Infantil la teoría pedagógica de Loris Malaguzzi (Malaguzzi, 2014) y la experiencia de Reggio Emilia (Fernández Santín \& Feliu Torruella, 2017; Lewin Benham, 2011), el aprendizaje significativo y su práxis (Ausubel, 2002), los paradigmas de calidad de la Educación Infantil (Azcona \& Hoyuelos, 2011), así como el decálogo de buenas prácticas en Educación Infantil formulado por Hoyuelos $(2013,2015)$, coordinador de las escuelas municipales de Pamplona, basado en que las propuestas de intervención en el aula tienen que estar en un marco teórico que ponga en valor el gran potencial de la infancia para construir su propio aprendizaje, en una escuela construida por toda la comunidad educativa; la práctica debe ir encaminada a educar, con docentes capacitados para ofertar propuestas interesantes y proyectos abiertos que faciliten un mayor conocimiento de la cultura de la infancia y proporcionen experiencias ricas, de interés para los niños y niñas. El papel del profesorado es el de observar, acompañar, aprender con la infancia y documentar los procesos de vida, respetando la cultura y los tiempos de los más pequeños. El espacio, interlocutor importante en el proceso educativo, debe ser cuidado y el material polisensorial, ofertado estéticamente.

A partir de este marco teórico, cada investigador/a elabora un listado de experiencias, el equipo establece un orden de prioridad, decide las que se analizarán cada año y se distribuyen entre los miembros del grupo. 


\section{Instrumento}

Para diseñar la entrevista inicial, único instrumento de la investigación, se toman las siguientes decisiones. Primero se define la temática: contexto de la práctica, su fundamentación teórica, evolución de la experiencia, impacto de la iniciativa y su transferibilidad. El contenido se organiza teniendo en cuenta los objetivos de la investigación: descripciones y valoraciones sobre la práctica, concepciones sobre creencias y teorías, y finalmente teorías prácticas sobre cómo deberían hacerse las cosas según el informante.

Los coordinadores de cada grupo, reunidos en Santiago, sede del investigador principal del proyecto, consensuan un primer diseño de preguntas en función de las cuatro categorías anteriores, que son sometidas a experimentación con diferentes informantes de distintos contextos educativos, y tras el análisis de las mismas y constatadas las deficiencias, se incorporan los reajustes necesarios. Finalmente se diseña el instrumento con ítems comunes para todos los investigadores y equipos de trabajo, que permite reconstruir el desarrollo profesional del profesorado, evidenciar los cambios que se han producido en cuanto a sus ideas en torno a la docencia y la Educación Infantil, y analizar la buena práctica. El modelo de registro contiene las siguientes cuestiones finales:

¿Podría hacer un relato de su biografía a modo de resumen?

¿Cuál fue su paso por la escuela, cómo la recuerda?

¿Cuáles han sido sus procesos formativos?

¿Qué momento de su formación tuvo más impacto en su proyecto de vida?

¿Qué momentos han sido especialmente importantes en su carrera? ¿Cuáles fueron las principales facilidades y las mayores dificultades? ¿Qué cosas cambiaría con respecto a sus experiencias de formación?

¿Desde cuándo realiza esta buena práctica? ¿Por qué la inició? ¿Cómo fue evolucionando?

¿Cómo está organizada? ¿De quién depende y quién la gestiona? ¿A quién va dirigida? ¿Es obligatoria? ¿Qué tiempo promedio le suele otorgar?

¿Qué coste tiene para el centro escolar o empresa?

¿Cómo se evalúa la buena práctica, qué se evalúa?

¿Sobre qué suele versar la buena práctica? ¿Por qué se escogen esas temáticas?

¿Cómo se lleva a cabo por unidades didácticas, por bloques temáticos, por proyectos?

¿Se evalúa el rendimiento de los participantes? ¿Cómo?

¿Incorpora las TICs y el e-learning?

¿Se han generado algunos materiales propios de qué tipo, estaría dispuesto a ceder imágenes de los mismos para poder enriquecer el trabajo de los demás docentes?

Cuénteme, por favor, alguna experiencia de buena práctica de la que esté más satisfecho o que le haya dejado un buen recuerdo.

¿Por qué considera importante esa buena práctica?

¿En su opinión qué condiciones se han de dar para que la buena práctica sea de calidad?

¿En general está satisfecho con la buena práctica que está siguiendo?

¿En qué cree que ha mejorado su empresa/organización a consecuencia de la buena práctica?

¿Ha hecho alguna evaluación del impacto real de la buena práctica, con qué resultados?

¿Produce alguna ventaja la buena práctica sobre las personas que la realizan?

En su opinión, ¿qué valor tiene la buena práctica dentro del centro/empresa? 
También en su opinión ¿qué están haciendo bien los centros escolares/empresas en cuanto a las actuaciones en Educación Infantil y que están haciendo mal? ¿Quisiera añadir alguna cosa más?

\section{Procedimiento de recogida y análisis de datos}

El estudio de cada caso se inicia explicando el proyecto a la institución responsable y se negocia la posibilidad de realizarlo. Si la respuesta es positiva se firma un acuerdo de ética y discrecionalidad en la investigación, junto con el compromiso de retorno del trabajo a la institución y se organiza la fase de recogida de información a través de la entrevista (instrumento) y los encuentros informales con los agentes de la iniciativa. El estudio se realiza in situ a través de la entrevista con los educadores y posteriormente, una vez finalizada la investigación y realizado el informe por los investigadores, se les entrega a los responsables para que lo analicen y enriquezcan con aportaciones sobre descripciones y valoraciones de la práctica, teniendo en cuenta las evidencias recopiladas por los docentes (no hay que olvidar que han sido seleccionados también por realizar investigaciones sobre su propia práctica educativa) y el sentido que tienen dentro de la experiencia. Durante el proceso se producen encuentros con los docentes para aclarar dudas y analizar la documentación institucional y profesional, generada por la práctica (registros de conversaciones y de documentación de la práctica, así como de las producciones de los niños). En esta última cita, se discute sobre aspectos contextuales de tipo social y cultural, ambiente físico en el que se realiza, espacios y recursos, participantes, etc. También sobre la secuencia de fases que lleva la acción desde una perspectiva global; las actividades realizadas qué se hace, dónde, con qué, con quién, con qué resultado y finalmente los roles de adultos y de infancia. Esto permite observar el pensamiento infantil, sus producciones y su desarrollo competencial como los argumentos que realizan para comprender sus experiencias (Mérida, González \& Olivares, 2017), que por su carácter confidencial obran en poder de los centros. Concluye el procedimiento con un encuentro para que el docente interprete y valore las evidencias y el sentido que tienen dentro de la experiencia.

Se emplea una estrategia cualitativa de análisis de contenido que permite tanto conocer enfoques y perspectivas de los docentes, su versión de lo que es y se hace en la experiencia analizada (descripciones y valoraciones), como reconocer las orientaciones globales del profesorado hacia la Educación Infantil (concepciones) y llevar a cabo un estudio detallado de sus ideas sobre aspectos concretos del cuidado y aprendizaje de los niños y niñas (teorías prácticas). El procedimiento adoptado para diseñar posibles categorías a las que asignar cada respuesta, después de transcribir las encuestas, fue el modelo de categorización «constant comparison method», estrategia ya utilizada por este grupo de investigación en el proyecto anterior sobre el conocimiento experto de los eméritos españoles, 2008-2012 y en el referente a los diarios de clase, bajo la denominación de "ideogramas". Se basa en que el equipo discute hasta establecer un criterio consensuado, y una vez alcanzado el «acuerdo interjueces» se clasifican las respuestas en una u otra categoría. El sistema de categorías, teniendo en cuenta las descripciones y valoraciones sobre la práctica, las concepciones sobre creencias y teorías, y finalmente las teorías prácticas sobre cómo deberían hacerse las cosas, se resuelve en tres y responden a: las características del profesorado, a los componentes teóricos y a los componentes prácticos que caracterizan la buena práctica. 
La unidad de estudio es la buena práctica, por lo que se realiza un primer análisis intra-práctica, un segundo intra-grupo por grupo o cluster de modalidad o área curricular, y un tercero inter-prácticas, como conjunto de buenas prácticas, con el objetivo de identificar y representar dinámicamente la estructura de características, principios y condiciones que caracterizan una buena práctica educativa, así como evidenciar la relación entre las creencias implícitas y las prácticas analizadas, empleando una metodología de orientación fundamentalmente cualitativa y etnográfica, que finaliza en un dispositivo informático que permita visualizar las ideas subyacentes en las prácticas estudiadas.

La fase de análisis permite visibilizar elementos clave de cada experiencia y las relaciones entre ellos, de tal forma que se identifica dinámicamente el esquema conceptual de los agentes y se relaciona con las secuencias de acción que se han desarrollado.

En suma, se identifican las características básicas (contextuales, conceptuales, operativas, actitudinales) de cada experiencia, se sistematizan las cualidades que la convierten en una buena práctica y se esquematizan y construyen los ejes interactivos de cada experiencia, relacionando fundamentación y puesta en práctica, ideas y acciones.

Tras este proceso cada investigador concluye el estudio del caso que le ha correspondido, presenta los resultados al grupo, se analiza el resultado comparándolo con el resto de las prácticas asumidas por el grupo y posteriormente con las del colectivo al finalizar cada uno de los años de la investigación, donde se vuelven a comparar las evidencias para dar fiabilidad a los resultados.

Durante este último año se está iniciando el análisis global de los diversos agrupamientos por modalidades de atención, áreas curriculares y niveles académicos, para sintetizar las características de las 100 experiencias estudiadas y elaborar el Informe final. El producto que se espera del proyecto es un repositorio de 100 buenas prácticas descritas y analizadas que se incorporarán a una WEB del equipo de investigación, y se pondrán a disposición de todas aquellas personas e instituciones vinculadas a la Educación Infantil y a las políticas de infancia, cumpliendo con ello uno de los objetivos estratégicos que proponían los organismos internacionales ya citados.

\section{Resultados}

De las evidencias recuperadas mediante el instrumento diseñado para tal fin, emerge un sistema de tres categorías con varios indicadores cada una de ellas, que permite marcar y representar las líneas de actuación que definen la buena práctica (Ver tabla 1).

Tabla 1

Sistema de categorías emergentes e indicadores que surge en el proceso de análisis de resultados

\begin{tabular}{lll}
\hline $\begin{array}{l}\text { Características del } \\
\text { profesorado }\end{array}$ & $\begin{array}{l}\text { Componentes teóricos } \\
\text { de la buena práctica }\end{array}$ & $\begin{array}{l}\text { Componentes prácticos } \\
\text { de la buena práctica }\end{array}$ \\
\hline $\begin{array}{l}\text { Curioso } \\
\text { Observador }\end{array}$ & $\begin{array}{l}\text { Sustentada en un marco } \\
\text { teórico sólido }\end{array}$ & $\begin{array}{l}\text { Surge de la iniciativa de la } \\
\text { infancia }\end{array}$ \\
Reflexivo & $\begin{array}{l}\text { Aporta mayor conocimiento } \\
\text { de la infancia }\end{array}$ & \begin{tabular}{l} 
Grupos pequeños \\
\hline
\end{tabular} \\
\hline
\end{tabular}




\begin{tabular}{|c|c|c|}
\hline \multirow{11}{*}{$\begin{array}{l}\text { Investigador } \\
\text { Escucha de forma activa } \\
\text { Comprometido con su } \\
\text { trabajo } \\
\text { Permanente formación } \\
\text { Creencias implícitas sobre } \\
\text { el alto potencial de la } \\
\text { infancia } \\
\text { Aprende junto a la infancia }\end{array}$} & \multirow{2}{*}{$\begin{array}{l}\text { Adaptada al momento } \\
\text { madurativo }\end{array}$} & Atención personalizada \\
\hline & & La infancia manipula y \\
\hline & \multirow{2}{*}{$\begin{array}{l}\text { Escenario educativo que } \\
\text { proporcione experiencias ricas } \\
\text { y reales }\end{array}$} & observa con todos los sentidos \\
\hline & & $\begin{array}{l}\text { Respeta los tiempos de la } \\
\text { infancia }\end{array}$ \\
\hline & $\begin{array}{l}\text { Entorno de confianza, respeto } \\
\text { y tolerancia }\end{array}$ & $\begin{array}{l}\text { Se desarrolla en pareja } \\
\text { educativa }\end{array}$ \\
\hline & \multirow{2}{*}{$\begin{array}{l}\text { Permite la investigación } \\
\text { abierta, con múltiples } \\
\text { respuestas }\end{array}$} & Participa toda la comunidad \\
\hline & & educativa \\
\hline & $\begin{array}{l}\text { Fomenta el pensamiento } \\
\text { divergente }\end{array}$ & $\begin{array}{l}\text { Materiales educativos } \\
\text { adecuados, ofertados } \\
\text { estéticamente }\end{array}$ \\
\hline & Desarrolla la creatividad & Exige tareas de \\
\hline & $\begin{array}{l}\text { Desarrolla estrategias de } \\
\text { pensamiento para interpretar } \\
\text { las experiencias }\end{array}$ & $\begin{array}{l}\text { documentación de los } \\
\text { procesos para analizar y } \\
\text { realizar debates pedagógicos }\end{array}$ \\
\hline & $\begin{array}{l}\text { Fomenta la autonomía } \\
\text { Desarrolla la autoestima } \\
\text { Lúdica }\end{array}$ & $\begin{array}{l}\text { Evaluación continua y abierta } \\
\text { a la comunidad educativa }\end{array}$ \\
\hline
\end{tabular}

Una buena práctica requiere de docentes con unos rasgos concretos, que pueden ser los siguientes: "gran compromiso con su trabajo, [...] curiosidad, ganas de aprender y de sorprenderse, $[\ldots]$ de hacer cosas diferentes, $[\ldots]$ con capacidad de reflexión y mentalidad investigadora [...], que escuchan de forma activa [...], observadores [...], entusiastas con su trabajo [...], en permanente formación [...], con gran respeto por la infancia". El docente debe estar convencido del alto potencial que tiene la infancia, "como un sujeto competente y no sometido a los deseos de las personas adultas [...] los niños pueden aprender muchas más cosas de las que pensamos, incluso sobre aspectos muy conceptuales, si lo adaptamos a su nivel [...]". Su papel en el proceso de enseñanza aprendizaje, es el de observar, escuchar de forma activa, acompañar y aprender a la vez que lo hace su alumnado, desde el asombro y el entusiasmo: "el centro es el niño y la actividad del docente surge de la observación y la escucha activa [...] yo en aquel momento jugaba y aprendía también con los niños [...]".

La buena práctica debe sustentarse en un corpus teórico sólido, "es fundamental poder apoyarla en un colchón teórico [...] es necesario conocer qué se ha hecho y hasta dónde se ha llegado, para sacarle el máximo partido [...] hay que investigar para conocer la literatura científica antes de implementar la práctica". Además, debe aportar mayor conocimiento de la infancia "tiene que ayudar a entender al niño y su manera de estar en el mundo [...] aprender a aprender lo que los niños nos están mostrando [...] ayuda a evidenciar capacidades infantiles que son difíciles de apreciar $[\ldots] "$

La buena práctica nace de una iniciativa activa de los pequeños, "fueron los niños y las niñas los que nos guiaron y orientaron en el descubriendo de este proyecto [...] surgió de los intereses e iniciativas que tienen [...] la motivación inicial es fundamental, y aunque sean pequeños tienen sus propios intereses, solo hay que descubrirlos". Además, es imprescindible que los niños disfruten mientras aprenden "Corrían, subían, bajaban $[\ldots]$ es fundamental que puedan experimentar y tengan la oportunidad de disfrutar [...] desde lo lúdico es como aprenden en estas edades". Debe proporcionar experiencias ricas, 
posibilitar la creatividad, el pensamiento divergente desde la investigación "que no tenga una única respuesta [...] es importante que el niño aprenda investigando [...] algo que parece inasequible se puede materializar en propuestas concretas [...] hay que ofrecer a los niños la posibilidad de investigar de entrar en el terreno de lo desconocido, en el mundo de la formulación de teorías e hipótesis, en la construcción de pruebas y experimentos para comprobarlas [...]". Debe servir para desarrollar estrategias de pensamiento que faciliten la comprensión de sus experiencias "es interesante observar cómo van construyendo los procesos y su mapa mental [...]". La buena práctica debe ser abierta a tantas respuestas como niños y lenguajes empleen "es importante que el docente sea flexible y no oponga resistencia a los cambios [...] son como descubrimientos que hacen ellos y tú ni te has planteado que van a surgir [...] primero observábamos, después analizábamos e introducíamos los cambios [...] no se puede ir pensando que lo controlas todo"

A nivel formal, debido a las características de la etapa y a la necesidad de documentar los procesos, los grupos deben ser pequeños "para poder observar y documentar la experiencia [...] en un grupo grande no se puede observar o experimentar [...] en grupos pequeños puedes prestar una atención mucho más personalizada [...] los pequeños necesitan actuar y experimentar y solo en grupos pequeños se puede conseguir que lo hagan [...] si trabajas en pareja educativa una va al taller con un grupo pequeño y la otra se queda con el grupo grande, esto ayuda a llevar a cabo las propuestas, a documentarlas y analizarlas con posterioridad [...]". Además, es importante tener en cuenta a toda la comunidad educativa "ser transparente, que la gente opine sobre lo que haces, por lo que debe tener en cuenta a toda la comunidad [...] es muy importante que todos estemos en el mismo barco y sepamos lo que estamos haciendo [...] toda la documentación la presentamos a las familias [...] es muy importante para la escuela la relación que se establece entre el entorno escolar y social [...] así mismo, contribuye a la relación que se establece entre el niño y el adulto, que debe ser emocionalmente fuerte y estable y a la que consideramos contribuye una buena práctica".

La buena práctica debe realizarse en un entorno de confianza y respeto, también a los tiempos de cada niño y niña "no debemos enseñar sino crear un entorno y unas condiciones para que sea la infancia la que quiera aprender [...] sin esperar productos finales [...] dándole tiempos largos, donde las cosas puedan pasar [...] respetar los tiempos de cada niño contribuir a crear un clima de respeto, libertad, participación y tolerancia [...] si el adulto tiene una actitud de escucha cercana al niño, se consigue que desarrolle su autonomía y su autoestima [...] no adelantarse a sus iniciativas, a sus deseos de participar y colaborar. La paciencia y el respeto de los tiempos de cada niño, es fundamental en su desarrollo". La buena práctica exige el empleo de materiales adecuados que inviten al juego integral "empleamos material polisensorial [...] preferiblemente materiales naturales [...] bien presentados". Además, tiene que estar adaptada a momento madurativo y atender al desarrollo integral de la infancia "hay que saber en qué momento evolutivo está cada niño [...] crear ambientes que faciliten la libertad de movimientos, la actividad autónoma, el descubrimiento de sus capacidades [...] es muy importante que puedan manipular y observar con todos los sentidos"

En el desarrollo de una buena práctica, son fundamentales las tareas de documentación de los procesos en reuniones pedagógicas "toda la documentación nos sirve para analizar y repensar la práctica, para ampliar la mirada e ir avanzando en el conocimiento [...] a 
través de la escucha activa, observación, documentación videográfica, reflexión y análisis de lo documentado, podemos comprender y profundizar sobre lo observado [...] realizamos cambios en la propuesta gracias a que documentamos los procesos [...]". Es fundamental la evaluación "se evalúan muchas cosas en las sesiones mediante la observación y la documentación según los criterios que hemos consensuado, qué tipo de relaciones se dan, a qué tipo de rincón acuden, durante cuánto tiempo, cómo abordan ese juego, qué materiales eligen, cómo los utilizan, qué les interesa [...] vienen otros profesionales a observarnos y a devolvernos qué es lo que han visto [...] acuden alumnas de la universidad en parejas un día a la semana y nos observan y luego nos reunimos con ellas y nos devuelven a modo de feb-back lo que han visto [...] mostramos la documentación a las familias [...] algunas familias acuden a observar a sus hijos y a participar con ellos en la experiencia y nos devuelven también sus impresiones [...] nuestra evaluación recoge muchos puntos de vista, el nuestro, el de las alumnas, las familias y el de otros profesionales".

\section{Discusión y conclusiones}

En la etapa de Educación Infantil se contempla un estudio internacional similar al que sustenta este artículo (Howes, 2010) situado en la línea estratégica de reconocer, analizar, normalizar, representar y dar a conocer buenas prácticas. De hecho en España son muy pocos los proyectos de investigación financiados que se han ocupado de analizar las dimensiones a tener en cuenta para satisfacer los criterios de buenas praxis, o de identificar las características que permiten atribuir esta categoría a una práctica, y solo el Grupo Interuniversitario de Estudios (GIE), se ha especializado durante los últimos años en el análisis de buenas prácticas a través de proyectos vinculados al Programa de Fomento de la Investigación Científica y Técnica de Excelencia (I+D).

Con respecto al primer y segundo objetivo del estudio, el instrumento diseñado para recopilar las evidencias y la metodología empleada en el análisis de las mismas, han permitido al grupo de trabajo, reconocer, analizar, normalizar, representar y hacer visibles un total de 12 buenas prácticas, 9 en el tramo 0-3 años, poniendo de relieve los diversos componentes conceptuales y operacionales que caracterizan una buena praxis en Educación Infantil.

Tanto las narraciones de las docentes implicadas, como las observaciones realizadas y los registros, han hecho posible rescatar información muy valiosa y ponerla al alcance de todo el profesorado mediante el empleo del mapa conceptual. Además, han facilitado establecer un marco teórico de características y condiciones plural y flexible sobre las experiencias analizadas, identificando qué tienen en común y de diferente, de propio y de transferible.

Se han identificado los ámbitos y los criterios teóricos básicos en los que se enmarca una buena praxis. Los elementos definitorios para observar si existen criterios de calidad en una práctica docente se han basado en última instancia en la innovación, transferibilidad, factibilidad, impacto positivo, planificación, responsabilidades y roles bien definidos, documentación y evaluación.

No sólo se han descrito, representado y visibilizado, sino que también se han extrapolado, permitiendo concluir sobre algunas categorías vinculadas a los fundamentos teóricos de la investigación sobre las buenas prácticas. 
Las maestras comparten unos rasgos personales y unas creencias asumidas sobre la escuela construida de forma conjunta entre todos sus miembros, donde hay coherencia entre las decisiones pedagógicas y económicas. Una escuela con un compromiso real de educar frente a enseñar, activa, investigativa, con espacios de reflexión, donde cada niño y niña es diferente y singular. Se considera que una buena praxis tiene que ser reconocida como tal por parte de las familias y de otros profesionales, y que además implica satisfacción profesional y personal, se ajusta a la naturaleza y a la cultura de la infancia, facilita un mayor conocimiento de las criaturas, responde a sus intereses, contempla todas sus potencialidades, sus estrategias de investigación para construir hipótesis y dar sentido a sus propias actuaciones, fomenta la curiosidad e interés de los niños y niñas, permite que construyan su propio aprendizaje mediante la experimentación y el juego, facilita el empleo de pluralidad de códigos interrelacionados para comunicarse, se realiza en pequeños grupos, propone experiencias reales, atractivas, estéticas y ajustadas a la infancia para conseguir procesos de cambio y desarrollos ricos, proporciona seguridad y placer por aprender, tanto a la infancia como al adulto y está abierta a nuevos enfoques. Las educadoras acompañan, observan, escuchan, investigan, documentan los proyectos, reflexionan, descubren, ahondan en las potencialidades del ser humano y dan un sentido humano a su profesión. Viven la práctica desde el interés, la complicidad, el asombro, lo inesperado, el respeto a la singularidad, a los intereses y a la autonomía de la infancia. En suma, aprenden de ella y junto a ella.

Además, las buenas experiencias educativas, en la línea de estudios afines precedentes, parten de la práctica diaria, de lo que sienten, vivencian y experimentan las educadoras y los niños. Surgen de la observación y de la posterior reflexión sobre la necesidad de responder al interés recurrente de los niños, y atienden a todos los aspectos implicados en el proceso. Exigen de formación específica y en su planificación se tienen en cuenta aspectos como el espacio, los recursos materiales y humanos, los grupos y la formación de las docentes. Requieren una reflexión sistemática y crítica sobre la propia praxis que se observa, se vivencia y se documenta de forma rigurosa; además se analizan, interpretan y discuten las evidencias en las reuniones pedagógicas, donde surgen las nuevas propuestas (Contreras \& Pérez de Lara, 2010; Piñero, 2014; Sennett, 2009), y se prima el buen hacer en la práctica (Zabalza, 2012).

Una buena praxis responde por tanto a los parámetros de mejora de la educación, ya que exige formación, investigación y reflexión sobre la práctica realizada y supone un cambio en la realidad educativa de la escuela (De Moya \& Madrid, 2015; Lewin Benham, 2011).

Se puede concluir que la metodología empleada, basada en las historias de vida (López de Maturana, 2012; Moriña, 2017) y la teoría del posicionamiento (Davies \& Harré, 1990), facilita la visibilización de las creencias asumidas epistemológicas, didácticas y actitudinales, por su importancia en la construcción de la identidad profesional (Barnett \& Di Napoli, 2007) y en las prácticas del profesorado. Permite a los docentes analizar la práctica desde los sentidos y las intenciones (Hoyuelos, 2015), descubrir los criterios que la estructuran e identifican, y evidenciar sus patrones de análisis y sus enfoques a la hora de definir y describir su actuación para dar sentido a su praxis (Zabalza, 1987).

En cuanto al tercer y último objetivo del trabajo, se apuesta por la oportunidad de generar a nivel nacional e internacional, grupos de trabajo que debatan sobre el modelo educativo, investiguen, reflexionen sobre la práctica de aula, evalúen los procesos y los 
resultados y faciliten a los profesionales de la Educación Infantil compartir experiencias de buenas praxis docentes. Además, los resultados de las investigaciones sobre la etapa, aparte de ser visibles a toda la comunidad educativa, deberían integrarse en los programas formativos de las universidades, contribuyendo así a mejorar la calidad educativa de los futuros docentes.

\section{Referencias}

Ausubel, D. (2002). Adquisición y retención del conocimiento: una perspectiva cognitiva. Barcelona: Paidós.

Azcona, J.M., \& Hoyuelos, A. (2011). Reflexiones sobre la calidad en las Escuelas Infantiles. Tarbiya. Revista de Investigación e Innovación Educativa, 42, 157-186. Recuperado de https://revistas.uam.es/tarbiya/article/view/273/259

Barnett, R., \& Di Napoli, R. (2007). Changing Identities in Higher Education. Londres: Routledge.

Bartau Rojas, I., Azpillaga Larrea, V. \& Joaristi Olariaga, L.M. (2017). Metodología de enseñanza en centros eficaces de la Comunidad Autónoma del País Vasco. Revista de Investigación Educativa, 35(1), 93-112. doi: http://dx.doi.org/10.6018/rie.35.1.225141

Bureau des institutions démocratiques et des droits de l'homme de l'OSCE [BIDDH] (2011). Education aux droits de l'homme dans les sytèmes scolaires d'Europe, d'Asiecentrale et d'Amérique du Nord. Recueil des bonnes pratiques. Varsovie: Bureau des institutions démocratiques et des droits del'homme de l'OSCE. Recuperado de http://www.osce.org/odihr

Bodero Cáceres, C. (2017). La neurociencia en la primera infancia. Apuntes de Ciencia $\mathcal{E}$ Sociedad, 7(1), 6-10. doi: http://dx.doi.org/10.18259/acs.2017002

Braslavsky, C. Abdoulaye, A., \& Patiño, M. I. (2003). Développement Curriculaire et «Bonne Pratique» en Éducation. Genève: Bureau International d'Education, UNESCO.

Campos, R. (2013). As indicações dos organismos internacionais para as políticas nacionais de educação infantil: do direito à focalização. Educação e Pesquisa, 39(1), 195-209. Recuperado de http://www.scielo.br/pdf/ep/v39n1/v39n1a13.pdf

Cid, A., Pérez, A., \& Zabalza, M. A. (2013). Las prácticas de enseñanza realizadas/ observadas de "los mejores profesores" de la Universidad de Vigo. Educación XX1, 16(2), 265-295. doi: https://doi.org/10.5944/educxx1.2.16.10342

Contreras, J., \& Pérez de Lara, N. (2010). Investigar la experiencia educativa. Madrid: Morata.

Davies, B., \& Harré, R. (1990). Positioning: the discursive production of selves. Journal for the Theoryof Social Behaviour, 20(1), 43-63. doi: https://doi.org/10.1111/j.1468-5914.1990.tb00174.x

De Moya, M. V., \& Madrid, D. (2015). La Educación Infantil que queremos: investigaciones y experiencias. Ensayos: revista de la Facultad de Educación de Albacete 30(2), 1-9. Recuperado de https://www.revista.uclm.es/index.php/ensayos/issue/view/106

Denébola, C., \& Rodríguez, J. (2016). Buenas prácticas en educación Infantil y materiales didácticos: Análisis de tres estudios de caso. En R. Romero (Coord.). Congreso Internacional de Innovación y tecnología educativa en Educación Infantil (pp. 1-18). Sevilla: Universidad de Sevilla.

Fernández- Santín, M., \& Feliu-Torruella, M. (2017). Reggio Emilia: An essential tool to develop critical thinking in early childhood. Journal of New Approaches in Educational Research, 6(1), 50-56. doi: https://doi.org/10.7821/naer.2017.1.207

Hoyuelos, A. (2013). Aprender en la escuela. Infancia: educar de 0 a 6 años, 137, 32-38.

Hoyuelos, A. (2015). Cuando la pedagogía se moja. Cuadernos de Pedagogía, 459, 88-95. 
Hoyuelos, A. (2017). Maddalena Tedeschi: Reggio Emilia: no en cualquier lugar. Cuadernos de Pedagogía, 475, 34-39.

Larose, F., Grenon, V., Bourque, J., \& Bédard, J. (2011). Análisis de la práctica docente y construcción de referente de competencias profesionales. Revista Española de Pedagogía, 69(248), 81-100. Recuperado de https://revistadepedagogia.org/wp-content/ uploads/2011/01/248-005.pdf

Lewin Benham, A. (2011). Twel ve Best Practices for Early Childhood Education. N. York DC: Teachers College Press.

López de Maturana, S. (2012). La profesionalidad docente desde las historias de vida de los buenos (as) profesores(as). Rizoma freireano, 12. Recuperado de http://www. rizoma-freireano.org/la-profesionalidad-docente-desde-las-historias-de-vida-de-losy-las-buenosas-profesoresas-silvia-lopez-de-maturana-luna

Malaguzzi, L. (2014). Els cent llenguatges dels infants. Infáncia: educar de 0 a 6 anyy, 200, 33-37.

Marcelo, C. (1987). El pensamiento del profesor. Barcelona: CEAC.

Mendioroz, A., \& Fiz, R. (2016). Creencias implícitas del profesorado emérito español: características de buenas praxis. Revista Electrónica de Investigación Educativa, 18(1), 183-196. Recuperado de http://redie.uabc.mx/redie/article/view/789

Mérida, R., González, E., \& Olivares, M.A. (2017). Estrategias y modalidades de argumentación oral en las asambleas de educación Infantil. Un estudio multi caso. Revista Complutense de Educación, 28(2), 445-462. doi: https://dx.doi.org/10.5209/rev_RCED.2017.v28.n2.49439

Mir Pozo, M.L., \& Ferret Ribot, M. (2014). Aproximación a la situación actual del profesorado de educación infantil. Revista electrónica interuniversitaria de formación del profesorado, 17(2), 235-255. doi: https://dx.doi.org/10.6018/reifop.17.2.181441

Molina, A., Miralles, P., Deusdad, B., \& Alfageme, M.B. (2017). Enseñanza de la historia, creación e identidades y prácticas docentes. Profesorado: revista de currículum y formación del profesorado, 21(2), 331-354. Recuperado de https://recyt.fecyt.es/index. php/profesorado/issue/view/3088.

Moriña, A. (2017). Investigar con historias de vida. Metodología biográfico-narrativa. Madrid: Narcea.

Novak, J. (1998). Conocimiento y aprendizaje. Los mapas conceptuales como herramientas facilitadoras para escuelas y empresas. Madrid: Alianza.

Piñero, M. (2014). Recursos para la práctica. Indicadores de buenas y malas prácticas. Aula de Infantil, 76, 25-27.

Sennett, R. (2009). El artesano. Barcelona: Anagrama.

Zabalza, M. A. (1987). Pensamiento del profesor y desarrollo didáctico. Enseñanza, Anuario Interuniversitario de Didáctica, 4(5), 109-13.

Zabalza, M. A. (2012). El estudio de las “buenas prácticas” docentes en la enseñanza universitaria. REDU Revista de Docencia Universitaria, 10(1), 17-42. Recuperado de https://dialnet.unirioja.es/ejemplar/312206

Zabalza, M. A. (2016). El Prácticum y las prácticas externas en la formación universitaria. Revista Prácticum, 1(1), 1-23. Recuperado de https://revistapracticum.com/ index.php/iop/article/view/15

Fecha de recepción: 18 de enero de 2018.

Fecha de revisión: 2 de febrero de 2018.

Fecha de aceptación: 22 de junio de 2018. 